\title{
A measurable entanglement criterion for two qubits
}

\author{
Hoshang Heydar: and Gunnar Björk \\ Department of Microelectronics and Information Technology, \\ Royal Institute of Technology (KTH), Electrum 229, SE-164 40 Kista, Sweden \\ Luis L. Sánchez-Soto \\ Departamento de Óptica, Facultad de Ciencias Físicas, Universidad Complutense, 28040 Madrid, Spain
}

(Dated: November 4, 2018)

\begin{abstract}
We propose a directly measurable criterion for the entanglement of two qubits. We compare the criterion with other criteria, and we find that for pure states, and some mixed states, it coincides with the state's concurrence. The measure can be obtained with a Bell state analyser and the ability to make general local unitary transformations. However, the procedure fails to measure the entanglement of a general mixed two-qubit state.

PACS numbers: $42.50 . \mathrm{Hz}, 42.50 . \mathrm{Dv}, 42.65 . \mathrm{Ky}$
\end{abstract}

\section{INTRODUCTION}

Entanglement, by which we mean the existence of nonlocal quantum correlations between spacelike separated systems, is one of the most fertile and thought-generating properties of quantum mechanics. It has become an essential resource for the new fields of quantum physics created in recent years 1, 2, 3, 4], with some potential applications such as quantum cryptography [5, 6] and quantum teleportation [7]. But the idea of quantum entanglement is not new, it goes back to the early days of quantum theory where it was initiated by Einstein, Podolsky and Rosen [8] and was later extended by Bell [9] in form of Bell inequalities.

During recent years, there has been ample activity to quantify the existence and the degree of entanglement. The quest has been directed to find measures that satisfy reasonable axioms, such as normalization, continuity, monotonicity, etc. [10], and that hopefully are simple, directly measurable, and valid for any quantum state in any dimension. The foundation was laid by Peres, who showed that a necessary criterion for separability was positivity of the density matrix upon partial transposition [1]. Soon thereafter, the Horodeckis proved that the criterion was also sufficient [12]. In principle, the criterion also showed a way to decide, experimentally, whether the members of an identical ensemble of bipartite states were separable or not. A direct test is out of the question, since partial transposition is a nonphysical process. However, to make an indirect test, one could first perform quantum tomography on the ensemble to reconstruct its associated density matrix. Subsequently, the positivity of the partial transpose of the estimated density matrix could be checked. A problem of this approach is that quantum state tomography is a resource-consuming task. Although it has been performed on a priori known states

*Electronic address: hoshang@imit.kth.se
13, 14, 15], extending the method to a priori unknown states, multipartite states, or highly excited states will be a difficult task. Neverteless, recently there has been an experimental demonstration of the method, where the polarization entanglement of two light beams was determined by state tomography [16]. In view of the experimental difficulties with (non-)separability tests via tomography, it is tempting to try to use Bell tests, but such schemes have been shown to fail, in general, since there exist classes of nonseparable states that do not violate any Bell inequality [17].

The result of Peres and Horodeckis spurred others to derive quantitative measures of entanglement for nonseparable states, such as entanglement of formation [18], distillable entanglement 19 , concurrence 20], or relative entropy of entanglement 21]. The problem with these measures, again, is that they are difficult to measure.

Other methods to measure the entanglement of unknown states have also been suggested. However for pure bipartite states it has been shown that there exists no single observable that can measure entanglement 23]. Optimal strategies involving local measurements on individual members of an identically prepared ensemble have been derived 23], as well as optimal measurements if one measures properties of the entire ensemble (coherent measurements) 25]. In both cases, it suffices to make local measurements on the two systems, but again, the measurements impose substantial experimental difficulties.

Entanglement witnesses is another method of detecting entanglement [26]. The witness operator is such that it gives a negative expectation value for its associated (class of) entangled state(s). The disadvantage with the witnesses is that a positive expectation value does not assure separability. Recently, entanglement witnesses that require only local measurements have been proposed [27, 28]. It is to be noted that entanglement witnesses have been experimentally implemented very recently [29].

Finally, a method that characterizes the entanglement for any bipartite state has been proposed [30]. This method is based on what is referred to as a structural 
approximation of the linear (antiunitary) transposition operator. Although, applicable to any bipartite state, the method is based on a POVM that seems difficult to implement experimentally.

In this paper, we develop a new measure for $2 \otimes 2$ bipartite quantum states, inspired by the measure of entanglement proposed in 22]. Our measure equals the concurrence for pure states and some classes of mixed states, and it is based on the bipartite phase sum and difference as quantified by a positive operator-valued measure (POVM) of quantum "phase". Although tailor-made POVMs (e.g., POVMS optimal for some purpose) are notoriously difficult to implement experimentally, it is shown that our measure can be obtained from a Bellstate analyzer, a single nonlocal observable. However, the measurement is supplemented by variable local unitary transformations, effectively rendering the measurement a multi-observable scheme. From the experimental viewpoint, such a measurement is, by comparison, relatively easy to implement.

\section{JOINT PHASE PROPERTIES OF TWO QUBITS}

As stated in 22], the most general POVM for a single qubit

$$
\begin{aligned}
\hat{\Delta}_{\gamma}(\varphi) & =\frac{1}{2 \pi}\left(\hat{I}+\gamma e^{i \varphi} \hat{S}_{+}+\gamma e^{-i \varphi} \hat{S}_{-}\right) \\
& =\frac{1}{2 \pi}\left(\hat{I}+\gamma e^{i \varphi}|1\rangle\left\langle 0\left|+\gamma e^{-i \varphi}\right| 0\right\rangle\langle 1|\right)
\end{aligned}
$$

where $0 \leq \gamma \leq 1$ to ensure positivity. It is easy to see that for any value of $\gamma$ we have $\int_{0}^{2 \pi} d \varphi \operatorname{Tr}\left[\hat{\rho}_{\mathrm{A}} \hat{\Delta}_{\gamma}(\varphi)\right]=1$, where $\hat{\rho}_{\mathrm{A}}$ is an arbitrary qubit density operator, so that $\operatorname{Tr}\left[\hat{\rho}_{\mathrm{A}} \hat{\Delta}_{\gamma}(\varphi)\right]$ is the probability distribution function of the qubit "phase" $\varphi$. Only the choice $\gamma=1$ enables the probability distribution to attain the value zero, so in the following we will assume that $\gamma=1$ and suppress this parameter in the notation. Suppose that we have two qubits, denoted A and B. It is now possible to form a POVM of this bipartite system by simply forming the tensor product

$$
\hat{\Delta}\left(\varphi_{\mathrm{A}}, \varphi_{\mathrm{B}}\right)=\hat{\Delta}_{\mathrm{A}}\left(\varphi_{\mathrm{A}}\right) \otimes \hat{\Delta}_{\mathrm{B}}\left(\varphi_{\mathrm{B}}\right),
$$

where, e.g., $\varphi_{\mathrm{A}}$ is the POVM "phase" associated with qubit A. We can now recast this POVM expressed in local properties in terms of the phase difference $\varphi_{-} \varphi_{\mathrm{A}}-\varphi_{\mathrm{B}}$ and sum $\varphi_{+} \varphi_{\mathrm{A}}+\varphi_{\mathrm{B}}$, both of which are joint properties of the qubits. Moreover, to make the associated POVM $2 \pi$-periodic we define [24]

$$
\begin{aligned}
\hat{\Lambda}\left(\varphi_{+}, \varphi_{-}\right)= & \frac{1}{2}\left[\hat{\Delta}\left(\frac{\varphi_{+}+\varphi_{-}}{2}, \frac{\varphi_{+}-\varphi_{-}}{2}\right)\right. \\
& \left.+\hat{\Delta}\left(\frac{\varphi_{+}+\varphi_{-}}{2}+\pi, \frac{\varphi_{+}-\varphi_{-}}{2}+\pi\right)\right]
\end{aligned}
$$

From this joint POVM, we can naturally form the associated joint probability distribution

$$
\mathcal{P}\left(\varphi_{+}, \varphi_{-}\right)=\operatorname{Tr}\left[\hat{\rho} \hat{\Lambda}\left(\varphi_{+}, \varphi_{-}\right)\right]
$$

where $\hat{\rho}$ is the two-qubit density operator. Next, we introduce measures of to what extent the properties of the bipartite system depend on the joint phase properties. To that [end] we compute

$$
\Gamma_{+}=\left|\int_{2 \pi} d \varphi_{+} e^{i \varphi_{+}} \mathcal{P}\left(\varphi_{+}, \varphi_{-}\right)\right|
$$

and

$$
\Gamma_{-}=\left|\int_{2 \pi} d \varphi_{-} e^{i \varphi_{-}} \mathcal{P}\left(\varphi_{+}, \varphi_{-}\right)\right|,
$$

which are the Fourier components of the joint phase probability distribution. Note that, due to the small size of the two qubit Hilbert space, $\mathcal{P}\left(\varphi_{+}, \varphi_{-}\right)$does not contain any Fourier components of higher frequency. Lastly, we define

$$
\Gamma=4 \pi\left|\Gamma_{+}-\Gamma_{-}\right|
$$

Computing this value explicitly for a two-qubit state, with its density operator $\hat{\rho}$ expressed in the standard qubit basis $|00\rangle,|01\rangle,|10\rangle,|11\rangle$, one obtains

$$
\Gamma=2|| \rho_{14}|-| \rho_{23}|| \text {. }
$$

This is our central result. From this equation one can immediately deduce that $0 \leq \Gamma \leq 1$. It is also clear that for any separable state, $\hat{\rho} \hat{\rho}_{\mathrm{A}} \otimes \hat{\rho}_{\mathrm{B}}$, one gets $\Gamma=0$ since $\rho_{14}=\rho_{A 12} \rho_{B 12}$ and $\rho_{23}=\rho_{A 12} \rho_{B 12}^{*}$. However, the measure $\Gamma$ is not invariant under local unitary transformations (and the measure $\mathbb{D}$ in 22 suffers from the same deficiency). This can easily be seen by looking at the Bell state $\left|\Phi^{+}\right\rangle=(|00\rangle+|11\rangle) / \sqrt{2}$. Its density matrix, expressed in the standard qubit basis, is

$$
\hat{\rho} \frac{1}{2}\left(\begin{array}{llll}
1 & 0 & 0 & 1 \\
0 & 0 & 0 & 0 \\
0 & 0 & 0 & 0 \\
1 & 0 & 0 & 1
\end{array}\right)
$$

from which one directly gets $\Gamma=1$, indicating two maximally entangled qubits. However, by making the local unitary transformation $|0\rangle \rightarrow(|0\rangle+|1\rangle) / \sqrt{2},|1\rangle \rightarrow$ $(|0\rangle-|1\rangle) / \sqrt{2}$ to qubit A only, the density matrix becomes

$$
\hat{\rho}=\frac{1}{4}\left(\begin{array}{cccc}
1 & 1 & 1 & -1 \\
1 & 1 & 1 & -1 \\
1 & 1 & 1 & -1 \\
-1 & -1 & -1 & 1
\end{array}\right)
$$

For this density matrix we get $\Gamma=0$ (and similarly, the measure $\mathbb{D}$ in $[22]$ is zero too, for this matrix). The reason for this result can loosely be stated as follows: We 
have based our measure on joint phase properties. However, there exists a complementary property to phase, which is excitation. (More precisely, relative phase, and relative excitation are complementary operators [31, 32]). The joint properties with respect to this complementary quantity are not probed by $\Gamma$. Hence, to get the complete information of the joint properties of the state, we need to make local transformations such that the entanglement, which is invariant under local unitary transformations, is manifested in the phase properties. Hence, we define our measure of entanglement $\Gamma_{\text {sup, }}$, where sup refers to the supremum of $\Gamma$ under all possible local unitary transformations. It follows directly from the definition that this measure is invariant of what local bases the density matrix is expressed in.

\section{AN EXPERIMENTAL IMPLEMENTATION}

In the remainder of this paper we shall compare it to some of the existing entanglement measures and we shall demonstrate how the measure can be experimentally measured. First, let us look at a pure two qubit state $|\psi\rangle=\alpha_{1}|00\rangle+\alpha_{2}|01\rangle+\alpha_{3}|10\rangle+\alpha_{4}|11\rangle$. For pure states, the concurrence, which is an established measure of entanglement, is $C=2\left|\alpha_{1} \alpha_{4}-\alpha_{2} \alpha_{3}\right|$ [20]. (The separability criterion $\alpha_{1} \alpha_{4}-\alpha_{2} \alpha_{3}=0$ has also been derived from a Hilbert space geometrical viewpoint in [33].) Our measure $\Gamma$, on the other hand, is $\Gamma=2|| \alpha_{1} \alpha_{4}^{*}|-| \alpha_{2} \alpha_{3}^{*}|| \leq C$. Equality is only achieved if either the complex vectors $\alpha_{1} \alpha_{4}$ and $\alpha_{2} \alpha_{3}$ are parallel, or if at least one of the coefficients $\alpha_{1}, \ldots, \alpha_{4}$ equals zero. Assume this is not the case. We then want to find $\Gamma_{\text {sup. }}$. To this end we perform local unitary transformations $\hat{U}_{\mathrm{A}} \otimes \hat{U}_{\mathrm{B}}=\hat{U}_{\mathrm{AB}}$ on the qubits $\mathrm{A}$ and $\mathrm{B}$. Assume that the transformation yields new state coefficients $\alpha_{1}^{\prime}, \ldots, \alpha_{4}^{\prime}$. The local transformations leave the concurrence $C$ invariant, so the relation $\Gamma \leq C$ still holds. If it is always possible to fulfill this relation with equality, then $\Gamma_{\text {sup }}=C$ for pure states. This is indeed always true. Assume, e.g., that we want to find a $\hat{U}_{\mathrm{AB}}$ that makes $\alpha_{1}^{\prime}=0$ (making $\Gamma=C$ ). The most general local unitary transformation will yield

$$
\begin{aligned}
\alpha_{1}^{\prime}= & \alpha_{1} \cos \varphi \cos \vartheta+\alpha_{2} e^{i \theta_{\mathrm{B}}} \cos \varphi \sin \vartheta \\
& +\alpha_{3} e^{i \theta_{\mathrm{A}}} \sin \varphi \cos \vartheta+\alpha_{4} e^{i\left(\theta_{\mathrm{A}}+\theta_{\mathrm{B}}\right)} \sin \varphi \sin \vartheta(11)
\end{aligned}
$$

where $\theta_{\mathrm{A}}$ and $\theta_{\mathrm{B}}$ are relative phases between the states in qubit $\mathrm{A}$ and $\mathrm{B}$, respectively, and the angles $\varphi$ and $\vartheta$ determine the mixing between the two states of each qubit. Choosing $\varphi=0, \vartheta \arctan \left|\alpha_{1} / \alpha_{2}\right|$, and $\theta_{\mathrm{B}}=$ $\arg \left(\alpha_{1} / \alpha_{2}\right)+\pi$ will ensure that $\alpha_{1}^{\prime}=0$ so that $\Gamma=$ $\Gamma_{\text {sup }}=C$. Hence, for all pure states our criterion gives identical results than the concurrence.

To find an experimental scheme to obtain our measure, we note that if we find some local unitary transformations that maximizes $\Gamma$, it is possible to simultaneously make $\rho_{14}$ and $\rho_{23}$ real by locally changing the relative phase between the qubit states. All such phase changes will leave $\left|\rho_{14}\right|$ and $\left|\rho_{23}\right|$ invariant. That is a transformation of the type

$$
\left(\begin{array}{cc}
1 & 0 \\
0 & e^{i \theta_{\mathrm{A}}}
\end{array}\right) \otimes\left(\begin{array}{cc}
1 & 0 \\
0 & e^{i \theta_{\mathrm{B}}}
\end{array}\right),
$$

can make $\rho_{14}$ and $\rho_{23}$ real and positive simultaneously. When this is the case, we note that $2 \rho_{14}=\left\langle\Phi^{+}|\hat{\rho}| \Phi^{+}\right\rangle-$ $\left\langle\Phi^{-}|\hat{\rho}| \Phi^{-}\right\rangle$, and $2 \rho_{23}\left\langle\Psi^{+}|\hat{\rho}| \Psi^{+}\right\rangle-\left\langle\Psi^{-}|\hat{\rho}| \Psi^{-}\right\rangle$. From these relations, the strategy becomes clear. We measure the state in a Bell-state analyzer and look at the difference between the detection probabilities $P_{\Phi^{+}}+P_{\Psi^{+}}-$ $P_{\Phi^{-}}-P_{\Psi^{-}}$. The maximum of this difference under all possible local unitary transformations of the state gives $\Gamma_{\text {sup. }}$ (We could, of course instead measure, e.g., $P_{\Phi^{+}}+P_{\Psi^{-}}-P_{\Phi^{-}}-P_{\Psi^{+}}$, as this function also reaches the maximum $\Gamma_{\text {sup }}$ when $\rho_{14}$ is real and positive and $\rho_{23}$ is real and negative. Hence, $\Gamma_{\text {sup }}$ is essentially the optimal entanglement visibility [34, 35]. Needless to say, such a measurement of entanglement would not be simple to perform on an unknown state, since we need to find the maximum over four continuous (but bounded) parameters. However, simulations we have made show that in most cases it suffices to maximize over each of the four parameters in turn, to find the global maximum. This is due to the fact that $\Gamma$ is a nicely behaved function for many classes of states. Making the local unitary transformations is relatively easy for, e.g., polarization-entangled photon pairs. In this case, the necessary transformations can be accomplished with geometric rotations and birefringent optics.

\section{MIXED STATES}

For mixed states the situation is more complicated and, in general, our measure will fail. We have already noted above that if the density matrix is a direct tensor product of any two qubit density matrices, then $\Gamma_{\text {sup }}=0$, as it should. The standard test of entanglement measures for mixed states is to look at the mixture of Bell, or Bell-like states. One such mixture, used by the Horodeckis [12], is the state

$$
\hat{\rho}=p\left|\psi_{1}\right\rangle\left\langle\psi_{1}|+(1-p)| \psi_{2}\right\rangle\left\langle\psi_{2}\right|
$$

where $0 \leq p \leq 1,\left|\psi_{1}\right\rangle=a|00\rangle+\sqrt{1-a^{2}}|11\rangle$ and $\left|\psi_{2}\right\rangle=$ $a|01\rangle+\sqrt{1-a^{2}}|10\rangle$. It is relatively straightforward to show that $\Gamma_{\text {sup }}=2 a\left(1-a^{2}\right)^{1 / 2}|1-2 p|$. That is, the state is nonseparable for $a \neq 0,1$ or $p \neq 1 / 2$. Another state that is often used for comparison is the Werner state

$$
\begin{aligned}
\hat{\rho}= & p\left|\Psi_{-}\right\rangle\left\langle\Psi_{-}\right|+(1-p)\left(\left|\Psi_{+}\right\rangle\left\langle\Psi_{+}\right|\right. \\
& \left.+\left|\Phi^{-}\right\rangle\left\langle\Phi^{-}|+| \Phi^{+}\right\rangle\left\langle\Phi^{+}\right|\right) / 3 .
\end{aligned}
$$

Again, it is not hard to compute that $\Gamma_{\text {sup }}=|1-2 p|$. That is, the state is separable for $p=1 / 2$, and maximally entangled for $p=1$. In both these cases our measure agrees with the concurrence. In fact, any state 
that can be written as a diagonal matrix in the Bell-basis $\left|\Phi^{+}\right\rangle,\left|\Phi^{-}\right\rangle,\left|\Psi^{+}\right\rangle,\left|\Psi^{-}\right\rangle$(with eigenvalues $\left.\lambda_{1}, \ldots, \lambda_{4}\right)$ will have the form

$$
\hat{\rho}=\frac{1}{2}\left(\begin{array}{cccc}
\lambda_{1}+\lambda_{2} & 0 & 0 & \lambda_{1}-\lambda_{2} \\
0 & \lambda_{3}+\lambda_{4} & \lambda_{3}-\lambda_{4} & 0 \\
0 & \lambda_{3}-\lambda_{4} & \lambda_{3}+\lambda_{4} & 0 \\
\lambda_{1}-\lambda_{2} & 0 & 0 & \lambda_{1}+\lambda_{2}
\end{array}\right)
$$

when written in the standard basis. From this we can easily compute that if two of the eigenvalues are zero, our measure $\Gamma_{\text {sup }}$ equals the concurrence $C$. If three or more eigenvalues are non-zero, our method fails, in general. Our method also fails for general mixed states of the form $\hat{\rho}=\sum_{n} P_{n}\left|\psi_{n}\right\rangle\left\langle\psi_{n}\right|$. Hence, unless one has some a priori information of the state, e.g., that it is of the class expressed in (14) or that it is pure, our proposed method is not reliable.

\section{DISCUSSION AND CONCLUSION}

In conclusion we have proposed a way of measuring the concurrence of any two-qubit pure state. An advan- tage with the method is that it effectively only requires one observable, although the state must be subjected to local unitary transformations before the measurement to determine its entanglement. A disadvantage compared to other proposed schemes is that the needed observable is nonlocal, meaning that one has to have access to both qubits. The method also works for some mixed states commonly used to test measures of entanglement, but fails for general mixed states. This serves as a warning that one has to be cautious when testing entanglement measures or measurement procedures with these states.

\section{Acknowledgments}

This work was supported by the Swedish Research Council (VR) and the Swedish Foundation for Strategic Research (SSF). The authors acknowledge useful discussions with Professor A. Karlsson and for helpful comments by Dr. P. Marsden.
[1] J. Preskill, Quantum Information and Computation, (California Institute of Technology, 1998).

[2] J. Gruska, Quantum Computing, (McGraw Hill, New York, 1999).

[3] M. A. Nielsen and I. L. Chuang, Quantum Computation and Quantum Information, (Cambridge University Press, Cambridge, 2000).

[4] M. Keyl, Phys. Rep. 369, 431 (2002).

[5] C. H. Bennett and G. Brassard, Proc. IEEE Int. Conference on Computers, Systems and Signal Processing (IEEE, New York, 1984); C. H. Bennett, F. Bessette, G. Brassard, L. Salvail, and J. Smolin, J. Cryptology 5, 3 (1992).

[6] A. K. Ekert, Phys. Rev. Lett. 67, 661 (1991).

[7] C. H. Bennett, G. Brassard, C. Crépeau, R. Jozsa, A. Peres, and W. K. Wootters, Phys. Rev. Lett. 70, 1895 (1993).

[8] A. Einstein, B. Podolsky, and N. Rosen, Phys. Rev. 47, 777 (1935).

[9] J. S. Bell, Physics 1, 195 (1964).

[10] M. J. Donald, quant-ph/0105017 v2.

[11] A. Peres, Phys. Rev. Lett. 77, 1413 (1996).

[12] M. Horodecki, P. Horodecki, and R. Horodecki, Phys. Lett. A 223, 1 (1996).

[13] D. T. Smithey, M. Beck, M. G. Raymer, and A. Faridani, Phys. Rev. Lett. 70, 1244 (1993).

[14] D. Liebfried, D. M. Meekhof, B. E. King, C. Monroe, W. M. Itano, and D. J. Wineland, Phys. Rev. Lett. 774281 (1996).

[15] A. I. Lvovsky, H. Hansen, T. Aichele, O. Benson, J. Mlynek, and S. Schiller, Phys. Rev. Lett. 87, 050402 (2001).

[16] M. Barbieri, F. De Martini, G. Di Nepi, and P. Mataloni, quant-ph/0307003 G. Di Nepi, F. De Martini, M.
Barbieri, and P. Mataloni, quant-ph/0307204

[17] R. F. Werner, Phys. Rev. A, 40, 4277 (1989).

[18] C. H. Bennett, D. P. DiVincenzo, J. Smolin, and W. K. Wootters, Phys. Rev. A 54, 3824 (1996).

[19] C. H. Bennett, H. J. Bernstein, S. Popescu, and B. Schumacher, Phys. Rev. A 53, 2046 (1996).

[20] W. K. Wootters, Phys. Rev. Lett. 80, 2245 (1998).

[21] V. Vedral, M. B. Plenio, M. A. Rippin, and P. L. Knight, Phys. Rev. Lett. 78, 2275 (1997).

[22] L. L. Sánchez-Soto, J. Delgado, A. B. Klimov, and G. Björk, Phys. Rev. A 66, 042112 (2002).

[23] J. M. G. Sancho and S. F. Huelga, Phys. Rev. A 61, 042303 (2000).

[24] A. Luis and L. L. Sánchez-Soto, Eur. Phys. J. D 3, 195 (1998)

[25] A. Acín, R. Tarrach, and G. Vidal, Phys. Rev. A 61, 062307 (2000).

[26] M. Lewenstein, B. Kraus, J. I. Cirac, and P. Horodecki, Phys. Rev. A 62, 052310 (2000).

[27] O. Gühne, P. Hyllus, D. Bruß, A. Ekert, M. Lewenstein, C. Macchiavello, and A. Sanpera, Phys. Rev. A 66, 062305 (2002).

[28] O. Gühne and P. Hyllius, Int. J. Theor. Phys. 42, 1001 (2003).

[29] M. Bourennane, M. Eibl, C. Kurtsiefer, H. Weinfurter, O. Guehne, P. Hyllus, D. Bruß, M. Lewenstein, and A. Sanpera, quant-ph/0309043

[30] A. K. Ekert, C. Moura Alves, D. K. L. Oi, M. Horodecki, P. Horodecki, and L. C. Kwek, Phys. Rev. Lett. 88, 217901 (2002); P. Horodecki and A. Ekert, Phys. Rev. Lett. 89, 127902 (2002); C. Moura Alves, P. Horodecki, D. K. L. Oi, L. C. Kwek, and A. K. Ekert, quant-ph/0304123

[31] A. Luis and L. L. Sánchez-Soto, Phys. Rev. A 48, 4702 
(1993).

[32] G. Björk, J. Söderholm, T. Tsegaye, and A. Trifonov, Phys. Scripta T102, 133 (2002).

[33] R. Mosseri and R. Dandoloff, J. Phys. A: Math. Gen. 34 10243 (2001).
[34] G. Jaeger, M. A. Horne, and A. Shimony, Phys. Rev. A 48, 1023 (1993).

[35] J. Voltz, C. Kurtsiefer, and H. Weinfurter, Appl. Phys. Lett. 79, 869 (2001). 